\title{
Indication of the Wigner threshold law in collisions between antiprotonic helium and hydrogenic molecules
}

\author{
B. Juhász ${ }^{a}$, D. Barna ${ }^{b}$, J. Eades $^{c}$, H. Fuhrmann ${ }^{d}$, \\ R.S. Hayano ${ }^{c}$, M. Horie ${ }^{e}$ D. Horváth ${ }^{a, b}$, H.A. Torii ${ }^{f}$, \\ E. Widmann ${ }^{d}$, H. Yamaguchi ${ }^{c}$, T. Yamazaki ${ }^{g}$, J. Zmeskal $^{d}$ \\ ${ }^{a}$ Institute of Nuclear Research, H-4001 Debrecen, Hungary \\ $b_{\text {KFKI RMKI, H-1525 Budapest, Hungary }}$ \\ ${ }^{c}$ Department of Physics, University of Tokyo, Japan \\ $d_{\text {Stefan Meyer Institute, A-1090 Vienna, Austria }}$ \\ ${ }^{e}$ CERN, CH-1211 Geneva 23, Switzerland \\ $f_{\text {Institute of Physics, University of Tokyo, Japan }}$ \\ $g_{\text {RI Beam Science Laboratory, RIKEN, Japan }}$
}

The temperature dependence of the quenching cross section of the state $(39,35)$ of antiprotonic helium in collisions with hydrogen and deuterium molecules has been measured. We found that the quenching cross section increases with decreasing temperature, in contradiction to the simple activation barrier model which could successfully describe the collisions in case of other antiprotonic states. The temperature (i.e. thermal velocity) dependence of the cross section roughly follows the $1 / v$ Wigner threshold law of exothermic reactions involving neutral particles.

\section{Introduction}

The lifetimes of the metastable states of antiprotonic helium atoms $\left(\overline{\mathrm{p}} \mathrm{He}^{+} \equiv \overline{\mathrm{p}}-\mathrm{e}^{-}-\mathrm{He}^{2+}\right.$, characterized by the principal quantum number $n$ and orbital quantum number $l$ ) decrease when $\overline{\mathrm{p}} \mathrm{He}^{+}$atoms collide with $\mathrm{H}_{2}$ or $\mathrm{D}_{2}$ molecules $[1,2,3]$. This quenching phenomenon is particularly interesting because i) the quenching remains strong even below $30 \mathrm{~K}$, ii) the 
quenching cross section $\sigma_{q}$ strongly depends on the quantum numbers $(n, l)$ of the $\overline{\mathrm{p}} \mathrm{He}^{+}$ atom $[4,5,6]$, and iii) $\sigma_{q}$ is usually a factor of $\sim 1.5$ smaller for $\mathrm{D}_{2}$ than for $\mathrm{H}_{2}[7,8]$.

From the physico-chemical point of view an antiprotonic helium atom can be thought of as an exotic hydrogen atom with a $\left[\overline{\mathrm{p}} \mathrm{He}^{2+}\right]_{(n, l)}$ 'nucleus' with an effective charge of $\sim 1.7$ e. Around this an electron is bound in a $1 s$ orbit with a binding energy of $\sim 25 \mathrm{eV}$. Thus by studying the $\overline{\mathrm{p}} \mathrm{He}^{+}+\mathrm{H}_{2}$ and $\overline{\mathrm{p}} \mathrm{He}^{+}+\mathrm{D}_{2}$ collisions we can obtain information on the reactions

$$
\begin{aligned}
& {\left[\overline{\mathrm{p}} \mathrm{He}^{+}\right]_{(n, l)}+\mathrm{H}_{2} \rightarrow\left[\mathrm{He}^{+} \overline{\mathrm{p}} \mathrm{H}\right]^{*}+\mathrm{H},} \\
& {\left[\overline{\mathrm{p}} \mathrm{He}^{+}\right]_{(n, l)}+\mathrm{D}_{2} \rightarrow\left[\mathrm{He}^{+} \overline{\mathrm{p}}\right]^{*}+\mathrm{D},}
\end{aligned}
$$

which are the most probable reactions responsible for quenching. The above reactions are very similar to the most fundamental hydrogen exchange reactions such as $\mathrm{D}+\mathrm{H}_{2} \rightarrow \mathrm{DH}$ $+\mathrm{H}$, whose cross sections are not known at low temperatures. This is very unfortunate since similar low temperature reactions play an important role in e.g. cold interstellar and protostellar clouds. In case of antiprotonic helium, however, the formed complex 'molecule' is expected to be short-lived $(\tau \lesssim \mathrm{ns})$, therefore the reaction is immediately followed by the annihilation of the antiproton. By detecting these annihilations, we can measure the lifetime and consequently the quenching cross section of an antiprotonic state. The molecular complex is most likely deeply bound so the reactions are exothermic.

According to the theoretical calculations of Sauge and Valiron [9], an $(n, l)$-dependent activation barrier exists for the $\overline{\mathrm{p}} \mathrm{He}^{+}+\mathrm{H}_{2}$ and $\overline{\mathrm{p}} \mathrm{He}^{+}+\mathrm{D}_{2}$ collisions, which can qualitatively explain the observed $(n, l)$ dependence of the quenching cross section $\sigma_{q}$. Based on the activation barrier model, the temperature $(T)$ dependence of $\sigma_{q}$ can be written as

$$
\sigma_{q}=\sigma_{0} \exp \left(-E_{b} / k T\right)+\sigma_{t},
$$

where the first term expresses an Arrhenius-type temperature dependence, while $\sigma_{t}$ accounts for a temperature-independent tunnelling effect. Here $E_{b}$ is the state-dependent barrier height, $\sigma_{0}$ is the cross section at infinitely high temperatures (pre-exponential cross section), and $k$ is the Boltzmann constant.

We already measured $\sigma_{q}$ of the antiprotonic states $(n, l)=(38,37)$ and $(37,34)$ at different temperatures, and concluded that the temperature dependence of $\sigma_{q}$ does follow Eq. (3) in case of these two states [10]: at higher temperatures $(T \gtrsim 100 \mathrm{~K})$, the Arrhenius term dominates, and $\sigma_{q}$ decreases with decreasing temperature. However, it levels off below $\sim 30 \mathrm{~K}$, where $\sigma_{t}$ is the dominating term. Now we made a detailed temperature scan of the state $(39,35)$ as well. Previously, this state has only been studied at $30 \mathrm{~K}$, where the quenching cross section was found to be close to the geometrical cross section, both with $\mathrm{H}_{2}$ and $\mathrm{D}_{2}$. This, and also the calculations of Sauge and Valiron, suggested that there is no activation barrier for the state $(39,35)$ and thus all collisions should result in quenching. Since there is no activation barrier, Eq. (3) no longer holds and one might expect a temperature-independent (i.e. constant) quenching cross section.

\section{Experimental setup and method}

The experimental setup was practically identical to our previous measurements $[10,11$, 12]. Antiprotonic helium atoms were created by stopping pulses of $\sim 3 \times 10^{7}$ antiprotons 
(arriving every $\sim 100$ seconds) with an energy of $5.3 \mathrm{MeV}$ in a cryogenic helium gas target. After slowing down by collisions with helium atoms, about $3 \%$ of the antiprotons are captured into various long-lived $(\tau \sim \mu \mathrm{s})$ metastable states. The subsequent annihilation of the captured antiprotons (mainly into charged pions) could be observed in two adjacent Cherenkov counters, which were connected to two gateable fine-mesh photomultipliers. The outputs of the photomultipliers were recorded using a digital oscilloscope as an 'analog delayed annihilation time spectrum' which is essentially the annihilation rate of the antiprotons versus time. A dye laser pumped by a Nd:YAG laser was used to irradiate our helium target after each antiproton pulse. The gas target consisted of ${ }^{4} \mathrm{He}$ (purity: 99.9996\%) to which hydrogen (purity: 99.999\%) or deuterium (purity: 99.8\%) was premixed at molar concentrations of 30 to $10000 \mathrm{ppm}$ with a relative accuracy of $2 \%$.

The quenching cross section of a metastable antiprotonic state can be obtained by measuring its decay rate $\gamma$ at different admixture (hydrogen or deuterium) concentrations. Assuming that the antiprotonic helium atoms are quenched in binary collisions with the admixture molecules, $\gamma$ can be expressed in terms of the state-dependent quenching cross section $\sigma_{q}$ as

$$
\gamma=\gamma_{0}+n_{\text {adm }} v_{\text {th }} \sigma_{q}
$$

where $\gamma_{0}$ is the 'intrinsic' decay rate of the state in a collision-free environment (this is calculated theoretically), $n_{\text {adm }}$ is the absolute number density of the admixture molecules, and $v_{\text {th }}=\sqrt{8 k T /\left(\pi M_{\text {red }}\right)}$ is the average relative thermal velocity, with $M_{\text {red }}$ being the reduced mass of the colliding species [13]. To obtain the decay rate $\gamma$ of a metastable state, we induced transitions between this state and a short-lived $(\tau \lesssim$ ns) state by a resonant laser pulse, and measured the antiproton population of the metastable state versus time. A detailed description of this so-called 't1-scan' method can be found in our previous publication [10].

\section{Results and discussion}

The left side of Fig. 1 shows the logarithm of the newly and previously $[6,7,10]$ measured quenching cross sections of the state $(39,35)$ versus the inverse temperature (Arrhenius plot). One can clearly see that $\sigma_{q}$ increases with decreasing temperature, both with $\mathrm{H}_{2}$ and $\mathrm{D}_{2}$. This contradicts the naive assumption that it should be temperature-independent and close to the geometrical cross section. In fact, at the lowest temperature, the measured $\sigma_{q}$ is almost twice as large as the geometrical cross section. The cross section of the state $(39,35)$ does not follow Eq. (3) either. For comparison, the right side of Fig. 1 shows $\sigma_{q}$ of the state $(38,37)$ measured previously $[6,10]$, which does follow Eq. (3).

One process that might cause the increase of the quenching cross section of the state $(39,35)$ at low temperatures is the following. Due to the lack of the activation barrier for the state $(39,35)$, the potential between the $\overline{\mathrm{p}} \mathrm{He}^{+}$atom and the colliding $\mathrm{H}_{2} / \mathrm{D}_{2}$ molecule is always attractive, therefore the $\mathrm{H}_{2} / \mathrm{D}_{2}$ molecule is attracted towards the $\overline{\mathrm{p}} \mathrm{He}^{+}$atom even from a distance, which results in an increased cross section. At lower temperatures, the velocity of the colliding species is lower, therefore this 'capture' process is enhanced.

Figure 2 shows the quenching cross section of the state $(39,35)$ with $\mathrm{D}_{2}$ as a function of the inverse collisional velocity $1 / v_{\text {th }}$. The data points are quite well aligned on a straight 

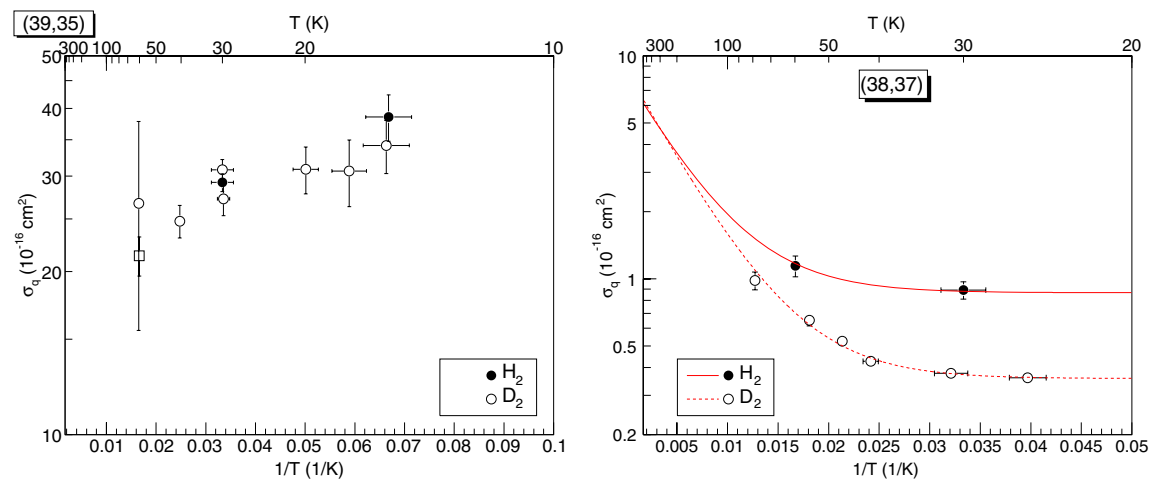

Figure 1: Left: Arrhenius plot of the newly and previously measured quenching cross sections of the state $(39,35)$. Right: for comparison, previously measured data for the state $(38,37)$. From [10].

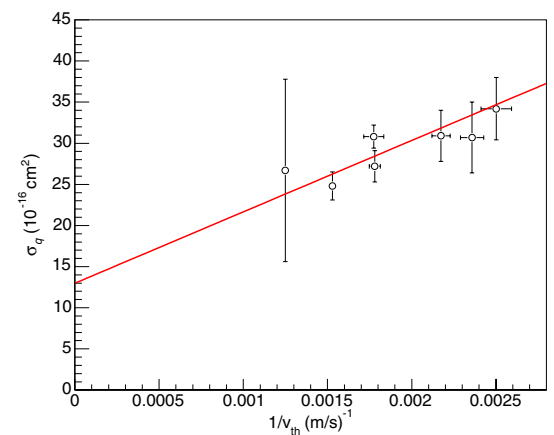

Figure 2: Quenching cross section $\sigma_{q}$ of the state $(39,35)$ with $\mathrm{D}_{2}$ as a function of the inverse collisional velocity $1 / v_{\text {th }}$.

line; the correlation factor of the points is 0.849 . Such a dependence of a cross section on the inverse velocity is consistent with the Wigner threshold law which states that in case of an exothermic reaction involving neutral particles near a threshold, the cross section is expected to follow an $1 / v$ dependence in the low temperature limit [14]. The result of a fit of a straight line is also drawn in Fig. 2. We had no time to measure the quenching cross section above $60 \mathrm{~K}$, therefore it is not known whether the cross section has a $1 / v$ dependence at high temperatures as well, or on the contrary, it levels off around the geometric cross section $\sigma_{\text {geom }}=21 \times 10^{-16} \mathrm{~cm}^{2}$.

Unfortunately, there are no theoretical calculations on the $\overline{\mathrm{p}} \mathrm{He}^{+}-\mathrm{H}_{2}$ or $\overline{\mathrm{p}} \mathrm{He}^{+}-\mathrm{D}_{2}$ system which would investigate the above-mentioned 'capture' mechanism and therefore it is not known whether it significantly increases the quenching cross section and if it does then 
whether it follows a $1 / v$ dependence. There are, however, calculations on similar systems. One calculation on the $\mathrm{H}+\mathrm{H}_{2}$ and similar reactions found that the rate constants $K \approx \sigma v$ of the $\mathrm{D}+\mathrm{H}_{2} \rightarrow \mathrm{DH}+\mathrm{H}$ and the $\mathrm{D}+\mathrm{DH} \rightarrow \mathrm{D}_{2}+\mathrm{H}$ exothermic exchange reactions are independent of the temperature below $\sim 10 \mathrm{~K}$ [15], and another calculation on the relaxation of vibrationally excited $\mathrm{H}_{2}$ molecules in collisions with ${ }^{3} \mathrm{He}$ and ${ }^{4} \mathrm{He}$ atoms found a $1 / v$ dependence of the cross section of this process below $\sim 30 \mathrm{~K}[16]$.

\section{Acknowledgements}

We are indebted to the CERN PS division for their efforts in operating the Antiproton Decelerator, and J.-M. Rieubland and his team of the CERN cryogenics laboratory for their help on our cryogenic system. We are grateful to Dr. S. Sauge and Dr. P. Valiron for the valuable discussions. This work was supported by the Grant-in-Aid for Specially Promoted Research (15002005) of Monbukagakusho of Japan, and the Hungarian Scientific Research Fund (OTKA T046095 and TeT-Jap-4/00).

\section{References}

[1] T. Yamazaki et al., Nature 361 (1993) 238.

[2] S.N. Nakamura et al., Phys. Rev. A 49 (1994) 4457.

[3] E. Widmann et al., Phys. Rev. A 53 (1996) 3129.

[4] T. Yamazaki et al., Chem. Phys. Lett. 265 (1997) 137.

[5] B. Ketzer et al., Phys. Rev. Lett. 78 (1997) 1671.

[6] B. Ketzer et al., J. Chem. Phys. 109 (1998) 424.

[7] B. Ketzer et al., Eur. Phys. J. D 13 (2001) 305.

[8] B. Juhász et al., Eur. Phys. J. D 18 (2002) 261.

[9] S. Sauge, P. Valiron, Chem. Phys. 283 (2002) 433.

[10] B. Juhász et al., Chem. Phys. Lett. 379 (2003) 91.

[11] M. Hori et al., Phys. Rev. Lett. 87 (2001) 093401.

[12] M. Hori et al., Nucl. Instrum. Methods A 496 (2003) 102.

[13] J.B. Hasted, Physics of atomic collisions, 2nd ed., Butterworth \& Co., London, 1972.

[14] E. P. Wigner, Phys. Rev. 73 (1948) 1002; see also D. W. Schwenke, D. G. Truhlar, J. Chem. Phys., 83 (1985) 3454.

[15] T. Takayanagi et al., J. Chem. Phys. 86 (1987) 6133.

[16] N. Balakrishnan, R. C. Forrey, A. Dalgarno, Phys. Rev. Lett. 80 (1998) 3224. 
